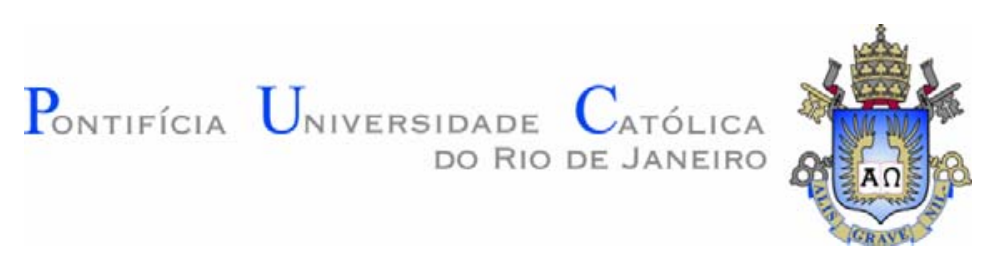

Eleazar Cristian Mejia Sanchez

Desenvolvimento de uma Máquina Tração-Torção de Ensaios de Fadiga para Avaliação de Modelos de Plasticidade Incremental

TESE DE DOUTORADO

DEPARTAMENTO DE ENGENHARIA MECÂNICA

Programa de Pós-Graduação em

Engenharia Mecânica

Orientador: Prof. Marco Antonio Meggiolaro

Rio de Janeiro, Maio de 2014 


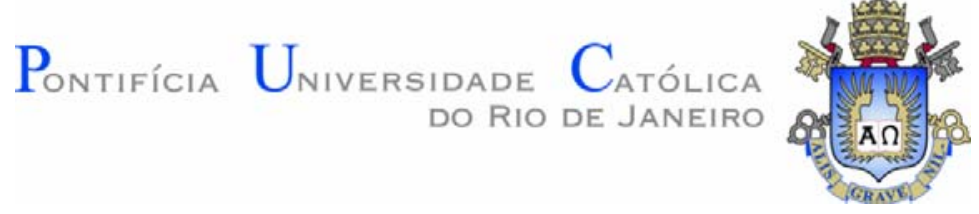

Eleazar Cristian Mejia Sanchez

\title{
Desenvolvimento de uma Máquina Tração-Torção de Ensaios de Fadiga para Avaliação de Modelos de Plasticidade Incremental
}

\begin{abstract}
Tese de Doutorado
Tese apresentada ao programa de Pós-graduação em engenharia Mecânica da PUC-Rio como requisito parcial para obtenção do título de Doutor em Engenharia Mecânica da PUC-Rio.
\end{abstract}

Orientador: Prof. Marco Antonio Meggiolaro

Volume I

Rio de Janeiro, Maio de 2014 


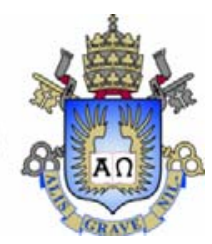

Eleazar Cristian Mejia Sanchez

\title{
Desenvolvimento de uma Máquina Tração-Torção de Ensaios de Fadiga para Avaliação de Modelos de Plasticidade Incremental
}

Tese apresentada como requisito parcial para obtenção do título de Doutor pelo Programa de Pós-Graduação em Engenharia Mecânica da PUC-Rio. Aprovada pela Comissão Examinadora abaixo assinada.

\author{
Prof. Marco Antonio Meggiolaro \\ Orientador \\ Departamento de engenharia Mecânica - PUC-Rio \\ Prof. Jaime Tupiassú Pinho de Castro \\ Departamento de engenharia Mecânica - PUC-Rio \\ Prof. Mauro Speranza Neto \\ Departamento de engenharia Mecânica - PUC-Rio \\ Profa. Deane de Mesquita Roehl \\ Departamento de Engenharia Civil - PUC-Rio
}

Prof. Nestor Zouain Pereira
UFRJ

Prof. Max Suell Dutra UFRJ

Prof. Timothy Hamilton Topper Waterloo University

Prof. José Eugenio Leal Coordenador(a) Setorial do Centro Técnico Científico - PUC-Rio 
Todos os direitos reservados. É proibida a reprodução total ou parcial do trabalho sem autorização da universidade, do autor e do orientador.

\section{Eleazar Cristian Mejia Sanchez}

Engenheiro Mecatrônico egressado da Universidad Nacional de Ingenieria (Peru), possui mestrado em Engenharia Mecânica pela Pontifícia Universidade Católica de Rio de Janeiro (PUC-Rio) com ênfase em Automação Industrial e Robótica

Ficha Catalográfica

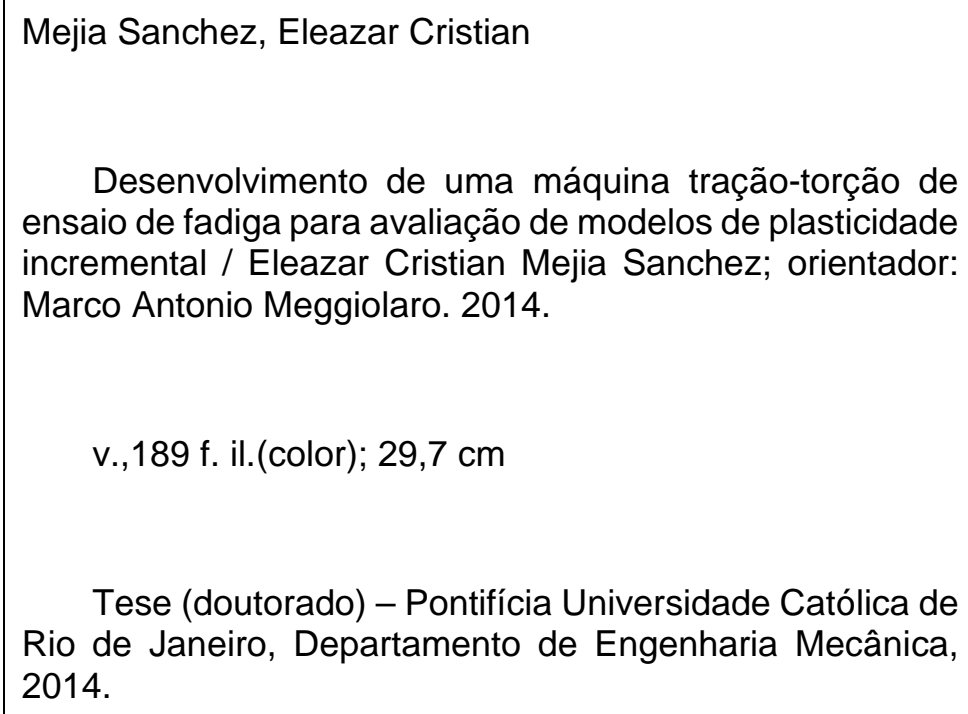

Desenvolvimento de uma máquina tração-torção de ensaio de fadiga para avaliação de modelos de plasticidade incremental / Eleazar Cristian Mejia Sanchez; orientador: Marco Antonio Meggiolaro. 2014.

v.,189 f. il.(color); 29,7 cm

Tese (doutorado) - Pontifícia Universidade Católica de Rio de Janeiro, Departamento de Engenharia Mecânica, 2014.

Incluí bibliografia.

1. Engenharia Mecânica - Teses. 2. Máquina de fadiga biaxial. 3. Fadiga Multiaxial. 4. Plasticidade Incremental. 5. Controle Sliding. 6. Célula de carga e torque. I. Meggiolaro, Marco Antonio. II. Pontifícia Universidade Católica do Rio de Janeiro. Departamento de Engenharia Mecânica. III. Título.

CDD: 621 
Ao senhor Jesus Cristo, meu pai Jesus Israel, minha mãe Agripina, meus irmãos Ronald e Liliana; e meus amigos. 


\section{Agradecimentos}

Ao Professor Marco Antonio Meggiolaro pela paciência e orientação durante o desenvolvimento do curso de doutorado.

Aos professores Jaime Tupiassú Pinho de Castro, Timothy Topper, José Luis Freire e Ronaldo Vieira pelos contínuos ensinamentos e lições de vida.

Ao Gerardo Castillo, Marco Perez, Jorge, Marco, Leonardo, Geancarlos, Jaiminho, Mourad amigos e colegas de laboratório.

A meu irmão Ronal, pelo apoio.

A todos as amizades que fiz durante o curso.

Aos professores da PUC - Rio pelo ensino.

Ao Departamento de Engenharia Mecânica da PUC - Rio e seus funcionários, pela colaboração comigo.

A CAPES Coordenação de Aperfeiçoamento de Pessoal de Nivel Superior pela ajuda financeira.

A FAPERJ Fundação Carlos Chagas Filho de Amparo à Pesquisa do Estado do Rio de Janeiro pela ajuda financeira.

A todas aquelas pessoas que de alguma outra forma participaram no desenvolvimento da tese. 


\section{Resumo}

Mejia Sanchez, Eleazar Cristian; Meggiolaro, Marco Antonio. Desenvolvimento de uma Máquina Tração-Torção de Ensaio de Fadiga para Avaliação de Modelos de Plasticidade Incremental. Rio de Janeiro, 2014. 189p. Tese de Doutorado - Departamento de Engenharia Mecânica, Pontifícia Universidade Católica do Rio de Janeiro.

A avaliação experimental de modelos de plasticidade incremental e a predição da vida à fadiga sobre cargas combinadas requer o uso de máquinas de testes multiaxiais. Neste trabalho é desenvolvida uma máquina tração-torção (MTT) para avaliar modelos de plasticidade incremental. Este sistema eletromecânico utiliza como atuadores principais dois motores de corrente contínua conectados a caixas de redução para gerar os carregamentos de tração axial e/ou torção. O projeto da MTT compreende a análise de sua integridade estrutural, seu dimensionamento, e o cálculo da vida à fadiga de seus principais componentes; o projeto e desenvolvimento de uma célula tração-torção (LTC - load torque cell); o desenvolvimento e implementação das técnicas de controle; e finalmente sua construção e avaliação. Uma técnica de controle PID por modos deslizantes (PID Sliding Mode control) foi especialmente desenvolvida para esta máquina, consistindo em aplicar um sinal de controle descontínuo que força o sistema a deslizar ao longo de uma superfície de convergência. Esta técnica de controle tem a capacidade de controlar continuamente a força axial e/ou o torque aplicado ao corpo de prova de maneira independente, o que permite gerar histórias de cargas não-proporcionais. Os métodos de controle são implementados em uma plataforma computacional em tempo real CompactRio. Deste modo, é possível gerar uma máquina de ensaios de fadiga multiaxial compacta, de fácil manuseio, que não precise de um sistema de controle complexo, e a um baixo custo. A máquina traçãotorção foi projetada para atender a uma ampla gama de ensaios de fadiga multiaxial, com uma capacidade de força axial máxima de \pm 200 kN e torque máximo de \pm 1300 N.m. O desempenho da MTT foi avaliado experimentalmente através de ensaios de plasticidade incremental. Para tanto, foram usados corpos de prova de tração-torção para medir seus comportamentos sobre cargas multiaxiais. Ensaios de encruamento não-proporcional, ratcheting (fluência cíclica) multiaxial e ratcheting uniaxial 
foram realizados em corpos de prova de aço inox 316, aço 1020, alumínio 7075 e alumínio 6063 na MTT, assim como em uma máquina Instron de 100 kN. Um simulador de plasticidade incremental para carregamentos de tração-torção foi desenvolvido, incorporando o modelo de encruamento cinemático não-linear de Jiang-Sehitoglu, e o modelo incremental de encruamento não-proporcional de Tanaka. Os parâmetros do material foram calibrados a partir de ensaios experimentais, permitindo que as simulações fossem capazes de prever o comportamento do material sobre diferentes histórias de carregamento, assim como as taxas de encruamento não-proporcional e de ratcheting. Os experimentos e simulações confirmaram tanto a adequabilidade da MTT desenvolvida, quanto do simulador de plasticidade incremental implementado, baseado nos modelos nãolineares de Jiang-Sehitoglu e Tanaka.

\section{Palavras-chave}

Máquina tração-torção de fadiga; Fadiga multiaxial; Plasticidade incremental; Controle por modos deslizantes; Célula de carga e torque. 


\section{Abstract}

Mejia Sanchez, Eleazar Cristian; Marco Antonio Meggiolaro (Advisor). Development of a Tension-Torsion Fatigue Testing Machine to evaluate incremental plasticity models, Rio de Janeiro, 2014. 189p. These of Doctor - Departamento de Engenharia Mecânica, Pontifícia Universidade Católica do Rio de Janeiro.

The experimental evaluation of incremental plasticity models and fatigue life prediction under combined loads requires the use of multiaxial testing machines. In this work, an axial-torsion machine (MTT) was developed to evaluate incremental plasticity models. This electromechanical system uses as a main actuators two DC motors connected to gearboxes to generate the axial and/or torsion loads. The design of axial-torsion machine comprises the analysis of its structural integrity, its dimensioning and fatigue life prediction its major components; the design and development of a load torque cell - LTC; the development and implementation of control techniques, and finally, its construction and its performance evaluation. A PID Sliding Model control technique has been specially developed for this machine, which consists in applying a discontinuous control signal that forces the system to slide along a surface convergence. This control technique has the ability to control the axial force and/or torsion applied to specimen test in an independent manner, which allows to generate a non-proportional loading histories. The control methods are implemented on a computing platform in real time CompactRio. Thus, it's possible to developed a compact multiaxial fatigue testing machine, easy to handle, which does not require a complex control system, and at a low cost. The tensiontorsion machine was designed to meet a wide range of multiaxial fatigue tests, with a maximum capacity of axial force of $\pm 200 \mathrm{kN}$ and torque of $\pm 1300 \mathrm{~N}$.m. The MTT performance was evaluated experimentally by incremental plasticity testing. For this purpose, tensile / torsion specimens were used to measure their behavior under multiaxial loads. Testing of non-proportional hardening, multiaxial ratcheting and uniaxial ratcheting (cyclic creep) were performed on specimens of 316 stainless steel, 1020 steel, 7075 aluminum and 6351T6 aluminum in the MTT, as well as a Instron Machine of $\pm 100 \mathrm{kN}$. A simulator of incremental plasticity to tensile-torsion loads has been developed, incorporating the non-linear kinematic hardening model of Jiang-Sehitoglu, and non-proportional hardening model of Tanaka. The material parameters were calibrated using experimental tests, allowing 
the simulations to predict the material behavior under different load histories, as well as rates of non-proportional hardening and ratcheting. The experiments and simulations confirmed both the suitability of the developed MTT, as well as the simulator of incremental plasticity implemented, based on non-linear models of Jiang-Sehitoglu and Tanaka.

\section{Keywords}

Tension-Torsion fatigue machine; Multiaxial fatigue; Incremental plasticity; Sliding control; Load torque cell. 


\section{Sumário}

1. Introdução 21

1.1. Objetivo 21

1.2. Considerações iniciais 21

1.3. Motivação 22

1.4. Revisão bibliográfica 23

1.5. Conteúdo da tese 28

2 Fundamentos de Plasticidade Incremental 30

2.1. Introdução 30

2.2. Algoritmos de plasticidade incremental 30

2.2.1. Tensor de tensão e deformação 31

2.2.2. Redução de ordem do espaço de tensão e deformação 34

2.2.3. Incremento de deformação plástica equivalente e deformação plástica total 38

2.2.4. Função de escoamento e regra de fluxo 39

2.2.5. Regra do fluxo plástico no espaço desviatório 5D 40

2.3. Modelos de plasticidade incremental 43

2.3.1. Encruamento cinemático 44

2.3.2. Encruamento isotrópico 45

2.3.3. Encruamento não-proporcional 46

2.3.4. Fluência cíclica ou Ratcheting 49

3 Simulador de Plasticidade Incremental 54

3.1. Introdução 54

3.2. Encruamento isotrópico incremental 54

3.2.1. Encruamento isotrópico baseado em deformação plana 54

3.3. Encruamento Não Proporcional Incremental 56

3.3.1. Modelo Generalizado de Tanaka 56

3.4. Modelo de encruamento cinemático múltiplas-superfícies 57

3.4.1. Representação de múltiplas-superfícies 58 
3.4.2. Regras da translação das superfícies

61

3.4.3. Descrição do algoritmo

4. Projeto Estrutural e Modelagem da Máquina Tração-Torção

67

4.1. Introdução

67

4.2. Projeto da MTT (biaxial)

67

4.2.1. Descrição geral da MTT

67

4.2.2. Modelo simplificado da MTT

69

5. Projeto e Integração dos Atuadores e Transdutores da MTT

71

5.1. Introdução

71

5.2. Desenvolvimento da célula de carga e torque

71

5.2.1. Projeto estrutural da LTC

71

5.2.2. Configuração e conexão dos extensômetros 80

5.2.3. Fabricação da LTC 85

5.3. Calibração dos transdutores da MTT 85

5.3.1. Calibração da célula de carga e torque 86

5.3.2. Calibração do LVDT- linear 88

5.3.3. Calibração do LVDT- rotação 89

5.4. Desenvolvimento do sistema experimental MTT 90

5.4.1. Conexões elétricas da MTT 91

5.4.2. Módulo de controle compactRio 92

5.4.3. Software desenvolvido em LabVIEW 94

6. Sistema de Controle 95

6.1. Introdução 95

6.2. Controle PID 96

6.2.1. Controle proporcional $(P) \quad 97$

6.2.2. Controle integral (I) 97

6.2.3. Controle derivativo (D) 98

6.2.4. Saída do controlador $\mathrm{u}(\mathrm{k}) \quad 99$

6.3. Controle por modos deslizantes " sliding mode control - SMC" 100

6.3.1. Superfície de deslizamento 100

$\begin{array}{ll}\text { 6.3.2. Lei de controle } & 102\end{array}$ 
6.3.3. O fenômeno de vibração "Chattering" 102

6.4. Aplicação da técnica de SMC na MTT 103

6.5. Resultados experimentais das técnicas de controle 105

7. Resultados Experimentais 116

7.1. Introdução 116

7.2. Resultados experimentais do modelo de fluência cíclica (ratcheting)

7.2.1. Determinação das propriedades do aço 1020 e alumínio $6351 T 6116$

7.2.2. Fluência cíclica (Ratcheting) uniaxial 120

7.2.3. Fluência cíclica (Ratcheting) multiaxial 128

7.3. Resultados experimentais do modelo de encruamento não proporcional 133

7.3.1. Determinação das propriedades do material 133

7.3.2. Levantamento de propriedades Al-7075 e aço inox 316

7.3.3. Encruamento não proporcional do Al-7075 139

7.3.4. Encruamento não-proporcional Aço Inox-316 144

8. Conclusões e Trabalhos Futuros 158

8.1. Conclusões 158

$\begin{array}{ll}\text { 8.2. Trabalhos Futuros } & 160\end{array}$

$\begin{array}{lr}\text { Referências Bibliográficas } & 161\end{array}$

$\begin{array}{ll}\text { Apêndice A } & 169\end{array}$

A.1 Determinação da força e momento da MTT 169

A.2 Analise da rigidez axial e torsional da MTT (biaxial) 171

$\begin{array}{ll}\text { A.2.1 Calculo da rigidez axial } & 171\end{array}$

A.2.2 Cálculo da rigidez torsional 180

A.3 Rigidez axial do modelo simplificado da MTT no Ansys. 185 


\section{Lista de figuras}

Figura 1.1. Ensaio de Fadiga $\quad 22$

Figura 1.2 Máquina Instron 8802TT para ensaios de fadiga multiaxial [17] 26

Figura 1.3 Máquina MTS tração torção modelo 370.02 [19] 26

Figura 2.1 A superfície de escoamento de Mises no plano $\left|\sigma_{x}-\sigma_{y}\right|$

com o vetor normal e a regra de fluxo plástico

Figura 2.2 Superfície de escoamento de Mises no espaço de tensão desviatório $6 \mathrm{D}$ e $5 \mathrm{D}$, onde ilustra-se o vetor normal $\overline{\mathrm{n}}$ e a regra de fluxo

Figura 2.3 Encruamento cinemático

Figura 2.4 Encruamento isotrópico para um material que encrua

Figura 2.5 Efeito da carga cíclica NP sobre o encruamento NP, e laços de histerese proporcional e NP causado pela mesma gama $\Delta \varepsilon$ num aço AISI 304

Figura 2.7 Ratcheting uniaxial para um material submetido a uma história de tensões desequilibrada entre $\sigma_{\max }>\mathrm{S}_{\text {Yc }} \mathrm{e}-\mathrm{S}$ Yc.

Figura 2.8 Ratcheting uniaxial para um material submetido a uma história de tensões desequilibradas com (a) alta e (b) baixa amplitude de deformação

Figura 3.1. (a) Superfície de escoamento, encruamento e falha no sub espaço $\sigma_{\mathrm{x}} \times \tau_{\mathrm{xy}} \sqrt{3}$ de $\mathrm{E}_{5 \mathrm{~s}} \mathrm{e}$ (b) raios correspondentes $\mathrm{r}_{\mathrm{i}}$ e o módulo de plasticidade generalizado $\mathrm{C}_{\mathrm{i}}$

Figura 3.2 Superfície de escoamento, encruamento e falha no sub espaço desviatório $S_{1} \times S_{2}$ para $M=3$, apresentando o vetor de translação da superfície de escoamento $\bar{\alpha}^{\prime}$

Figura 3.3 Configurações (a) sem encruamento e (b) saturado das superfícies consecutivas i e i+1

Figura 4.1 Modelo Inicial da Máquina Tração Torção

Figura 4.2 Modelo simplificado da MTT

Figura 5.1 Seção circular oca da estrutura da LTC

Figura 5.2 Estado de tensões típico através do Círculo de Mohr 72

Figura 5.3 Estado de deformações típicos, através do Círculo de Mohr 
Figura 5.4 Deformações da LTC analisadas no Ansys. 76

Figura 5.5 Esquema geral da LTC 76

Figura 5.6 a) Perfil melhorado do entalhe b) Comparação de $K_{t}$ do

perfil com r constante, e melhorado

Figura 5.7 Cálculo do fator de concentração de tensão no entalhe melhorado 77

Figura 5.8 Ponte de Wheatstone 80

Figura 5.9 Seção central da LTC 82

Figura 5.10 Conexão dos extensômetros na LTC, como célula de carga 83

Figura 5.11 Conexão dos extensômetros na LTC como célula de carga 84

Figura 5.12 Conexão dos extensômetros na LTC 85

Figura 5.13 Sistema de calibração da célula de carga 86

Figura 5.14 Curva de Calibração da LTC - célula de carga 87

Figura 5.15 Curva de Calibração da LTC - célula de torque 87

Figura 5.16 Sistema de calibração do LVDT Linear DT-100A 88

Figura 5.17 Calibração do LVDT Linear DT-100A 88

Figura 5.18 Calibração do LVDT Linear DT-100A para rotação 89

Figura 5.19 Calibração do LVDT rotação DT-100A 90

Figura 5.20 MTT desenvolvida no Laboratório de Fadiga - PUC-Rio 91

Figura 5.21 Esquema de conexões elétricas e do sistema de controle 91

Figura 5.22 Controlador $C R I O-9004 \quad 92$

Figura 5.23 Arquitetura interna do módulo FPGA 93

Figura 5.24 a) módulo NI-9263 e b) módulo NI-9237 94

Figura 5.25 Interação dos ambientes de programação do controlador 94

Figura 6.1 Sistema de controle em malha fechada 95

Figura 6.2 Resposta ao degrau do sistema 96

Figura 6.3 Diagrama de Blocos de um controlador PID 99

Figura 6.4 Superfície de deslizamento (Adaptado de Slotine e Li, 1991) 101

Figura 6.5 O fenômeno de Chattering (Adaptado de Slotine e Li, 1991) 103

Figura 6.6 Esquema do controle de força da MTT 103

Figura 6.7 Esquema geral do sistema de controle da MTT 105

Figura 6.8 Controle por modos deslizantes para um carregamento

tração de $\pm 30 \mathrm{kN} \quad 105$

Figura 6.9 Interface de controle no ambiente RealTime 106 
Figura 6.10 Controle por modos deslizantes para um

carregamento de $\pm 50 \mathrm{kN}$

Figura 6.11 Controle de torque por modos deslizantes para

$\begin{array}{ll}\text { torque de } \pm 71,6 \text { N.m } & 108\end{array}$

Figura 6.12 Controle PID sliding para um torque solicitado de \pm 71,6 N.m 108

Figura 6.13 Controle PID sliding para um torque solicitado de \pm 140 N.m 109

Figura 6.14 Controle de força ( $\pm 20 \mathrm{kN})$ e do torque

desejado $\left( \pm 71,6\right.$ N.m) $90^{\circ}$ fora de fase

Figura 6.15 a) Tensão normal $\sigma$ e cisalhante $\tau \sqrt{3}$ b) Gráfico $\sigma_{x} \mathrm{e}$ $\tau_{x y} \cdot \sqrt{3} 90^{\circ}$ fora de fase

Figura 6.16 Controle de força $( \pm 50 \mathrm{kN})$ e do torque desejado ( \pm 189 N.m) $90^{\circ}$ fora de fase

Figura 7.10 a) Tensão normal $\sigma=107 \mathrm{MPa}$ e cisalhante

$\tau \sqrt{3}=107 \mathrm{MPa}$ b) Gráfico $\sigma$ e $\tau \sqrt{3} 90^{\circ}$ fora de fase

Figura 7.1 Curva $\sigma_{x} x \varepsilon_{x}$ (a) Alumínio 6351T6 e (b) Aço 1020

117

Figura 7.2 Ajuste do coeficiente H e do expoente h do Al-6351T6

Figura 7.3 Curva monotônica do Al-6351T6

Figura 7.4 Curva monotônica do Aço 1020

Figura 7.5 Laço estabilizado, sob o controle de deformação de $\Delta \varepsilon= \pm 2 \%$. 120

Figura 7.6 Condição inicial para o ensaio de ratcheting uniaxial 121

Figura 7.7 Comportamento de fluência cíclica (ratcheting) do aço 1020. 122

Figura 7.8 Laços de histerese gerados por ratcheting uniaxial em aço 1020. 122

Figura 7.9 Deformação de ratcheting acumulada do aço 1020.

Figura 7.10 Taxa de deformação de ratcheting do aço 1020.

Figura 7.11 Laço de histerese estabilizado do Alumínio 6351T6 124

Figura 7.12 Condição inicial para o ensaio de ratcheting uniaxial do

Al-6351T6

Figura 7.13 Ensaio de ratcheting uniaxial Al-6351T6 com $\sigma_{a}=261 \mathrm{MPa} . \quad 126$

Figura 7.14 Deformação de ratcheting acumulada para o Al-6351T6 126

Figura 7.15 Taxa de deformação de ratcheting para $\sigma_{a}=261 \mathrm{MPa} \quad 127$

Figura 7.16 Ensaio de ratcheting uniaxial do Al-6351T6 com $\sigma_{a}=285 \mathrm{MPa} 127$ 
Figura 7.17 Deformação de ratcheting uniaxial acumulada

Figura 7.18 Taxa de incremento da deformação de ratcheting

Figura 7.19 Corpo de prova Tubular ratcheting multiaxial

Figura 7.20 Determinação das propriedades do Alumínio 6351Tx

Figura 7.21 Deformação cisalhante gerada pelo Torçor cíclico

Figura 7.22 Comportamento da deformação axial - Ratcheting multiaxial

Figura 7.23 Incremento da deformação axial para

$\sigma_{\mathrm{x}}=430 \mathrm{MPa}$ e T $=10 \mathrm{~N} . \mathrm{m}$

Figura 7.24 Deformação cisalhante gerado pelo $T= \pm 20$ N.m

Figura 7.25 Incremento da deformação axial para T = 20 N.m

Figura 7.26 Incremento da deformação axial para

$$
\sigma_{\mathrm{x}}=430 \mathrm{MPa} \text { e } \mathrm{T}=20 \mathrm{~N} \cdot \mathrm{m}
$$

Figura 7.27 Ajuste dos parâmetros de encruamento cíclico do Al-7075 134

Figura 7.28 Curva $\sigma \times \varepsilon \in$ cíclica do Al-7075 ajustando os bicos dos laços. 134

Figura 7.29 Laços estabilizados do Alumínio 7075.

Figura 7.30 Determinação dos parâmetros $E$ e $v$ do alumínio 7075.

Figura 7.31 Ajuste dos parâmetros de encruamento cíclico do Aço Inox 316.

Figura 7.32 Curva $\sigma \times \varepsilon$ cíclica do Aço inox-316 ajustando os

bicos dos laços.

Figura 7.33 Laços estabilizados do Aço inox 316.

Figura 7.34 Determinação dos parâmetros $E$ e $v$ do aço Inox-316.

Figura 7.35 Forma e dimensões do corpo de prova - ensaio

não proporcional.

Figura 7.36 Ensaio EN do Al-7075 $\operatorname{com} \varepsilon_{x}=0,2 \%$ a) laço histerese

$\sigma_{x}$ vs $\varepsilon_{x}$, b) laço histerese $\tau_{x y}$ vs $\gamma_{x y}$, c) trajetória das tensões

$\sigma_{x}$ vs $\tau_{x y} \cdot \sqrt{3}$, d) trajetória de deformação $\varepsilon_{x}$ vs $\gamma_{x y} / \sqrt{3}$.

Figura 7.37 Ensaio EN do Al-7075 $\operatorname{com} \varepsilon_{x}=0,4 \%$, a) laço histerese

$\sigma_{x}$ vs $\varepsilon_{x}$, b) laço histerese $\tau_{x y}$ vs $\gamma_{x y}$, c) trajetória das tensões

$\sigma_{x}$ vs $\tau_{x y} \cdot \sqrt{3}$, d) trajetória de deformação $\varepsilon_{x}$ vs $\gamma_{x y} / \sqrt{3}$.

Figura 7.38 Trajetória de deformação $\varepsilon_{x}$ vs $\gamma_{x y} / \sqrt{3}$ no ensaio NP 
do alumínio 7075 para uma deformação de $\varepsilon_{x}=0,2 \%$ e $\varepsilon_{x}=0,4 \%$

Figura 7.39 Ensaio EN do Inox-316 com $\varepsilon_{x}=0,1 \%$, a) trajetória

das tensões $\sigma_{x}$ vs $\tau_{x y} \cdot \sqrt{3}$, b) laço histerese $\sigma_{x}$ vs $\varepsilon_{x}$, c) laço histerese $\tau_{x y}$ vs $\gamma_{x y}$, d) trajetória de deformação $\varepsilon_{x}$ vs $\gamma_{x y} / \sqrt{3}$.

Figura 7.40 Ensaio EN do Inox-316 com $\varepsilon_{x}=0,25 \%$, a) trajetória

das tensões $\sigma_{x}$ vs $\tau_{x y} \cdot \sqrt{3}$, b) laço histerese $\sigma_{x}$ vs $\varepsilon_{x}$, c) laço histerese $\tau_{x y}$ vs $\gamma_{x y}$, d) trajetória de deformação experimental $\varepsilon_{x}$ vs $\gamma_{x y} / \sqrt{3}$,

e) trajetória de deformação simulada $\varepsilon_{x}$ vs $\gamma_{x y} / \sqrt{3}$, f) trajetória de deformação estabilizada, experimental e simulada. Figura 7.42 Ensaio EN do Inox-316 com $\varepsilon_{x}=0,31 \%$, a) trajetória das tensões $\sigma_{x}$ vs $\tau_{x y} \cdot \sqrt{3}$, b) laço histerese $\sigma_{x}$ vs $\varepsilon_{x}$, c) laço histerese $\tau_{x y}$ vs $\gamma_{x y}$, d) trajetória de deformação experimental $\varepsilon_{x}$ vs $\gamma_{x y} / \sqrt{3}$, e) trajetória de deformação simulada $\varepsilon_{x}$ vs $\gamma_{x y} / \sqrt{3}$,f) trajetória de deformação estabilizada experimental e simulado.

Figura 7.43 Trajetória de deformação $\varepsilon_{x}$ vs $\gamma_{x y} / \sqrt{3}$ no ensaio de encruamento NP do Aço inox-316 com $\varepsilon_{x}=0,1 \%, \varepsilon_{x}=0,25 \%$ e $\varepsilon_{x}=0,31 \%$.

Figura 7.44 Trajetória de deformação $\varepsilon_{x}$ vs $\gamma_{x y} / \sqrt{3}$ no ensaio de encruamento NP do Aço inox-316 com $\varepsilon_{x}=0,1 \%, \varepsilon_{x}=0,25 \%$ e $\varepsilon_{x}=0,31 \%$

Figura A.1 Tensão de cisalhamento a) tubo circular e b) eixo circular em torção

Figura A.2 Corpo de prova de tubular

Figura A.3 Modelo simplificado da MTT

Figura A.4 Modelo deformado da MTT pela força trativa

Figura A.5 Separação das vigas e colunas da MTT submetidas à tração

Figura A.7 (a) Modelo simplificado da MTT no Ftools e 
(b) deflexão da MTT, devido à carga trativa

Figura A.8 Simulações do modelo simplificado da MTT (a) Tensão e

(b) deflexão da MTT, devido à carga trativa

178

Figura A.9 Deformação na direção " $y$ " do modelo simplificado

da MTT no Ansys

179

Figura A.10 Modelo simplificado da MTT sobre Torção 180

Figura A.11 Modelo deformado da MTT pela Torção.

181

Figura A.12 Separação das vigas e das colunas da MTT

submetido à torção.

181

Figura A.13 Deslocamento dos extremos da MTT submetido a torção. 182

Figura A.14 Rotação da viga AB e CD da MTT submetido à torção.

182

Figura A.15 Modelo simplificado da MTT (a) Tensão e

(b) deflexão da MTT devido ao torçor

Figura A.16 Deformação na direção " $z$ " do modelo simplificado

da MTT gerado pelo torçor no Ansys

184

Figura A.17 Tensões no modelo simplificado da MTT devido à

carga trativa.

185

Figura A.18 Deslocamento na direção " $x$ " do modelo simplificado

da MTT

186

Figura A.19 Análise da falha por flambagem da MTT em

compressão pura

186

Figura A.20 Tensões no novo modelo da MTT devido a torção pura.

187

Figura A.21 Deslocamento na direção " $z$ " do novo modelo da MTT

188

Figura A.22 Análise da falha por flambagem da MTT em torção pura

188

Figura A.23Tensões de Mises devido a carregamento combinado

sobre a MTT

189 


\section{Lista de tabelas}

Tabela 1. Transformação direta e inversa entre o espaço 6D e 5D, na forma matricial

Tabela 2. Transformação direta e inversa entre o espaço 6D e 5D, na forma escalar

Tabela 3. Coeficiente de Encruamento NP.

Tabela 5. Constantes do material utilizadas para o projeto LTC

75

Tabela 5. Influência da espessura na vida à fadiga da LTC

79

Tabela 6. Sensibilidade mecânica e elétrica e faixa de forças da LTC

80

Tabela A.1 Influência do diâmetro na rigidez axial e torsional

185 